\title{
Anabases
}

ANABASES Traditions et réceptions de l'Antiquité

19 | 2014

Varia

\section{Moses I. Finley (1912-1986) et sa réception en}

\section{France}

Introduction

\section{OpenEdition}

Journals

Édition électronique

URL : http://journals.openedition.org/anabases/4754

DOI : 10.4000/anabases.4754

ISSN : 2256-9421

\section{Éditeur}

E.R.A.S.M.E.

\section{Édition imprimée}

Date de publication : 1 avril 2014

Pagination : 11-14

ISSN : $1774-4296$

Référence électronique

"Moses I. Finley (1912-1986) et sa réception en France », Anabases [En ligne], 19 | 2014, mis en ligne le 01 avril 2017, consulté le 21 octobre 2019. URL : http://journals.openedition.org/anabases/4754 ; DOI : 10.4000/anabases.4754 
Anabases 19 (2014), p. 11-14

\section{Moses I. Finley (1912-1986) et sa réception en France Introduction}

La RÉCEPTION FRANÇAISE de l'œuvre de Moses I. Finley a été importante et rapide. Les plus âgés d'entre nous l'ont accompagnée. À partir de la publication de la traduction du Monde d'Ulysse, en 1969, il est rapidement devenu un familier, quelqu' un qui, pour ainsi dire, allait faire partie de la famille du Centre dirigé par Jean-Pierre Vernant, qui s'appelait encore Centre de recherches comparées sur les sociétés anciennes, avant de prendre le nom de Centre Louis Gernet. Il était l'historien lu et enseigné, une référence et, bientôt, une autorité, mais il était plus : une personne à qui on pouvait s'adresser, qui répondait avec une grande ponctualité quand on lui envoyait un article ou un ouvrage, qu'on pouvait aller visiter ; bref une sorte d'oncle de Cambridge.

Les plus jeunes ont encore lu du Finley au cours de leurs études. Et, assurément, celles et ceux qui ont engagé des travaux sur Homère, l'esclavage ou l'économie, peutêtre la politique, n'ont pas manqué de le lire de plus près, pour comprendre ce qu'il avait écrit, comment il l'avait écrit et, si possible, pourquoi. Il leur fallait reconstituer les débats qui avaient alors cours et en saisir les enjeux. En ce sens, la réception se poursuivait et se poursuit : autres sont les questions, et autres, vraisemblablement aussi, ses points aveugles. Malgré tout, une visite aujourd'hui, dans une librairie à Paris, Cambridge, Londres, ou Chicago montre que ses livres ne sont plus très présents.

Publication d'une journée d'étude organisée par François Hartog (EHESS, CRH), Pauline Schmitt Pantel (université Paris 1 Panthéon-Sorbonne, ANHIMA), Alain Schnapp (université Paris 1 Panthéon-Sorbonne, ARSCAM), le 15 décembre 2012, à Paris. 
Grâce à la journée d'étude, qui s'est tenue à Paris en décembre 2012, nous avons désormais une vision plus précise de sa réception en France. Se vérifie aussi la règle qui veut qu'il n'y ait pas de réception sans malentendus, sans une part au moins de malentendus. Et c'est encore plus vrai quand il s'agit d'ouvrages conçus dans un autre pays, écrits dans une autre langue et pour un public peut-être différent. Si l'écart est trop grand entre ce qui est proposé, d'un côté, par l'auteur et ce qui est attendu, de l'autre, par des lecteurs, il n'y a pas de réception. Dans le cas d'un livre étranger, c'est très simple, nul n'a l'idée de le faire traduire. Or, Finley a été traduit. S'il a fallu quinze ans pour que paraisse Le monde d'Ulysse, ensuite les traductions se sont succédé rapidement jusqu'à son dernier livre, en 1987.

Mais de quels malentendus parle-t-on ? De ceux que Marshall Sahlins nommait des «malentendus productifs" (working misunderstandings) : de ceux qui font travailler les cultures, mobilisent leurs ressources pour légitimer une position, soutenir une action, produire du sens. En introduisant cette notion, il cherchait, en effet, à rendre compte au plus près de la rencontre tout à la fois bien réelle et manquée entre les Anglais et les Polynésiens à Hawaï. L'histoire est bien connue. Certes, Finley n'est pas Cook, et nous ne sommes pas des Hawaïens ; nous ne l'avons jamais pris pour le dieu Lono, mais nous ne l'avons pas mangé non plus ! L'affaire, plus modeste, de sa réception, se joue sur un tout autre terrain - celui du monde académique, celui d'une discipline, avec ses règles de production et de validation des énoncés - et à un tout autre moment - les années 1960 en France. Reste que la notion de malentendu productif pourrait servir à analyser en finesse les modalités de la réception ou, mieux, des réceptions de l'œuvre de Finley.

Comment a-t-elle commencé ? Au témoignage de Claude Mossé, qui évoque sa première rencontre avec lui, à l'occasion du congrès d'Aix-en-Provence de 1962 sur l'histoire économique, s'ajoutent les comptes rendus de Louis Gernet du premier livre de Finley sur les Horoi hypothécaires et une conférence donnée par ce dernier à Paris, en 1959, à l'Institut de droit romain, sur " La condition des non-libres dans la Grèce ancienne ». C'est ainsi le tout premier Finley, celui d'avant l'exil, comme le rappelle Riccardo Di Donato, qui a d'abord été reçu dans le petit milieu des juristes de l'Institut du Panthéon, alors dirigé par Henri Lévy-Bruhl. On retrouve le rôle de Gernet dans le passage du premier au deuxième Finley, celui du World of Odysseus (1954), puisqu'il en donne un notable compte rendu favorable dans l'Année sociologique, en 1956. Relevons, en passant, qu'il voit le meilleur du livre dans le dernier chapitre "Mœurs et valeurs ", qu'il lit comme " une précieuse contribution à une psychologie historique ».

Vient ensuite, en 1965, repère majeur et clairement identifié, "Économie et société en Grèce ancienne, l'œuvre de Moses I. Finley ». L'étude de Pierre VidalNaquet, il vaut la peine de le noter, est publiée, non dans une revue d'histoire ancienne ou même d'histoire générale, mais dans les Archives européennes de sociologie. Pourquoi ? Parce qu'elle lui avait été demandée par le directeur de la revue, Éric de Dampierre, un sociologue passé à l'ethnologie, qui souhaitait un papier sur Finley et Homère. C'est donc qu'il avait lu The World of Odysseus ou, au moins, la mise au point que Vidal- 
Naquet avait publiée, en 1963, dans les Annales: "Homère et le monde mycénien " ? Vidal-Naquet lui remit un texte d'une bonne cinquantaine de pages où le monde d'Ulysse n'est qu'une des rubriques ! Il venait de faire le même coup, l'année précédente, pour ce qui ne devait être que l'" avant-propos » du livre de Karl Wittfogel, Le Despotisme oriental; il le réitèrera à nouveau plus tard. Quoi qu'il en soit, le lecteur non antiquisant découvre un Finley complet, qui est déjà ou aussi "son » Finley, celui qui sera au cœur du manuel, conçu et rédigé avec Michel Austin, Économies et sociétés en Grèce ancienne. Publié en 1972, il est bien connu que le livre contribua à la formation de générations d'étudiants d'histoire.

La parution de la traduction du Monde d'Ulysse, due à la fille de Vernant, Claude Blanc, marque un autre temps fort. Le livre sort en 1969, chez Maspero. On le trouve en bonne place à "La Joie de Lire ", la librairie des éditions Maspero. Passer chez "Masp » était alors un itinéraire obligé. Combien d'exemplaires en ont été volés ? En tout cas, la publication à cette date et en ce lieu fait définitivement sortir Finley du cercle des seuls antiquisants. L'anthropologie est alors au plus haut, le livre est lu comme une contribution, non plus à la psychologie historique, mais à une anthropologie historique qui monte en puissance. Enfin, un historien de l'Antiquité, professeur à Cambridge de surcroît, qui parle aux autres historiens, qui est vraiment un collègue ! En 1965, Vernant avait publié chez Maspero Mythe et pensée chez les Grecs et, en 1968, le choix de textes de Gernet, sous le titre à valeur de slogan Anthropologie de la Grèce antique. Vidal-Naquet dirige une collection d'histoire classique. Finley vient renforcer la crédibilité de Maspero dans le domaine de l'histoire ancienne. Il est un renfort de poids pour les hérétiques.

Un dernier exemple, dans le cadre de cette introduction, d'usages inventifs de Finley est apporté par la publication de Démocratie antique et démocratie moderne en français, en 1976. Dans ses Mémoires, Vidal-Naquet écrit : "Finley m’avait demandé d'actualiser, pour le public français, un essai largement dirigé contre les apologistes américains de l'apathie politique "(Mémoires, 2, 1998, p. 233). Il se débarrasse de la tâche, presque du pensum, en quelques pages, pour arriver au plus vite à ce qui commence à l'intéresser vraiment. "Puisqu'il s'agit ici de la démocratie, remontons donc à la première grande expérience démocratique de l'Occident moderne, à la Révolution française et à ce qui l'a préparée, pendant l'époque des Lumières » (p. 15). Et voilà ! Cette fois, avec l'aide de Momigliano, est ouvert le champ des usages de l'Antiquité. Des analyses similaires pourraient être menées pour chaque livre traduit ou à l'occasion des visites de Finley en France. Pour être pleinement convaincantes, elles devraient prendre également en compte les contextes anglais et américain, pour mesurer les écarts et apprécier le potentiel de "malentendus productifs». Mais ces quelques exemples, ici évoqués plus que déployés, indiquent une voie que le lecteur pourra aisément parcourir et prolonger, en prenant connaissance des riches contributions qui suivent.

Enfin, le dossier réuni fait place à un Finley antérieur à toute réception, à peu près inconnu, celui du temps où Finley s'appelait Finkelstein, celui de ses années d'activisme 
et de militantisme, qui ont été aussi celles de sa formation intellectuelle et politique. À ce titre, elles ont préparé son travail ultérieur, quand il aura pris la décision de revenir vers le monde des études. Si bien qu'elles ont joué un rôle, ni immédiatement ni directement dans ce que nous avons appelé les malentendus productifs, mais, au moins, dans l'inscription de leurs possibilités. Nous devons cette enquête précise et inédite à Daniel Tompkins ${ }^{1}$.

1 Nos vifs remerciements vont à celles et ceux qui ont, d'une manière ou d'une autre, apporté leur concours à l'organisation et au bon déroulement de cette rencontre : contributeurs, intervenants, participants, ainsi qu'aux institutions suivantes : l'École des hautes études en sciences sociales, la Fondation Maison des sciences de l'homme, le Centre anhima, l'université Paris 1 Panthéon-Sorbonne. Nous remercions aussi le comité de rédaction de la revue Anabases d'avoir accepté de publier ce dossier et d'en avoir assuré l'édition scientifique. 